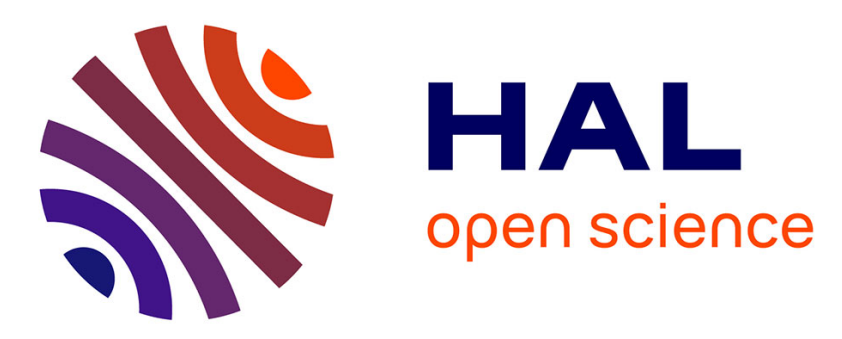

\title{
Estimating Localization Uncertainty Using Multi-hypothesis Map-Matching on High-Definition Road Maps
}

Franck Li, Philippe Bonnifait, Javier Ibañez-Guzmán

\section{- To cite this version:}

Franck Li, Philippe Bonnifait, Javier Ibañez-Guzmán. Estimating Localization Uncertainty Using Multi-hypothesis Map-Matching on High-Definition Road Maps. 20th IEEE International Conference on Intelligent Transportation Systems (ITSC 2017), Oct 2017, Yokohama, Japan. pp.1-6, 10.1109/ITSC.2017.8317804 . hal-01629119

\section{HAL Id: hal-01629119 \\ https://hal.science/hal-01629119}

Submitted on 6 Nov 2017

HAL is a multi-disciplinary open access archive for the deposit and dissemination of scientific research documents, whether they are published or not. The documents may come from teaching and research institutions in France or abroad, or from public or private research centers.
L'archive ouverte pluridisciplinaire HAL, est destinée au dépôt et à la diffusion de documents scientifiques de niveau recherche, publiés ou non, émanant des établissements d'enseignement et de recherche français ou étrangers, des laboratoires publics ou privés. 


\title{
Estimating Localization Uncertainty Using Multi-hypothesis Map-Matching on High-Definition Road Maps
}

\author{
Franck $\mathrm{Li}^{1,2}$, Philippe Bonnifait ${ }^{1}$ and Javier Ibanez-Guzman ${ }^{2}$ \\ ${ }^{1}$ Sorbonne universités, Université de Technologie de Compiègne, CNRS, Heudiasyc UMR 7253, \\ CS 60319, 60203 Compiègne \\ ${ }^{2}$ Renault s.a.s., Guyancourt, France. \\ Emails: \{franck.li,philippe.bonnifait\}@ @ds.utc.fr, javier.ibanez-guzman@ renault.com
}

\begin{abstract}
Lane-level road maps are necessary for Advanced Driving Assistance Systems (ADAS) and autonomous vehicles, providing prior contextual information on the road network. At this level of detail, map-matching becomes complex: much ambiguity can arise due to the high number of likely hypotheses (especially in dense urban areas) and due to the varying performance of GNSS positioning systems. This paper presents a method measuring the confidence in the information provided by the positioning system using a metric called Map Aided Horizontal Uncertainty Level (MA-HUL). It relies on a mapmatching algorithm designed to keep all likely hypotheses that are coherent with the vehicle odometry. Additionally, exteroceptive information is compared to the map data to remove ambiguities (i.e. lane marking detection from a camera). Some hypotheses are then trimmed if a mismatch is detected with sufficient confidence, keeping their number to a minimum. The use of a reference frame linked to the road is also studied to represent uncertainty independently in the along-track and cross-track axes. The performance of the proposed approach is illustrated using data recorded from an experimental vehicle operating on public roads.
\end{abstract}

\section{INTRODUCTION}

Intelligent vehicles functionalities improve continuously: most of modern Advanced Driving Assistance Systems (ADAS) require high accuracy positioning in order to perform correctly. But as the main automotive source of absolute positioning is Global Navigation Satellite Systems (GNSS), the accuracy obtained can vary greatly depending on the environment (especially in urban environments [1]). Systems relying heavily on positioning must therefore be able to quantify the confidence to give to the system: solutions have been developed based on Fault Detection and Exclusion (FDE) principle [2] but these require access to raw measurements in a tightly-coupled system.

A method using the computed position has been developed previously [3], providing a confidence metric called MapAided Horizontal Uncertainty Level (MA-HUL). It uses a multi-hypotheses lane-level map-matching algorithm based on a particle filter (PF) tuned to keep all likely hypotheses. This map-matching (MM) method relies mainly on car's odometry to find suitable positions on a high-definition road map (i.e. lane-level description of the road network with high accuracy) [4]. This method minimizes the role of the GNSS information, returning matching hypotheses using mostly dead reckoning (DR) and the map, the goal being to have a solution as independent of GNSS as possible, to get redundancy.

This paper improves previous work by using additional information from exteroceptive sensors, such as lane marking detection from a camera: comparing these to a priori data from the HD map can provide discriminating information (i.e. relative in-lane lateral position), allowing some ambiguities removal that could not be resolved without exteroceptive inputs. This additional information creates an asymmetry between the lateral and longitudinal components of the MA-HUL. It is therefore important to separate the metric in two values, considering distinct cross-track and an along-track MA-HUL.

This paper is structured as follows: Section II gives an overview of the multi-hypotheses map-matching used. The process of integrating the lane detection to the matching algorithm is developed in Section III. Section IV presents the MA-HUL as an integrity metric proposal. Section V shows the benefits of separating the MA-HUL into cross-track and alongtrack dimensions. Finally Section VI presents experimental results, evaluating the method.

\section{SEQUENTIAL MAP-MATCHING}

The map-matching algorithm used in this paper is described in details in [4]. The following gives a brief description of the method.

\section{A. Lane Level Map}

The road map used in this research is a mesoscale lanelevel road map [5] (see Fig. 1). This scale, situated between the macroscale (e.g. road guidance maps) and microscale (e.g. dense point cloud from perception sensors), is the most suitable for intelligent vehicles as it bears accurate information without being too dense to be easily used. The prototype map used in this paper, made by a mapmaker, covers $4 \mathrm{~km}$ of public roads in Compiègne, France with an absolute accuracy of $2 \mathrm{~cm}$. The map can thus be qualified of High-Definition map (HD map). It is built on a SQLite database containing the following relevant information: 


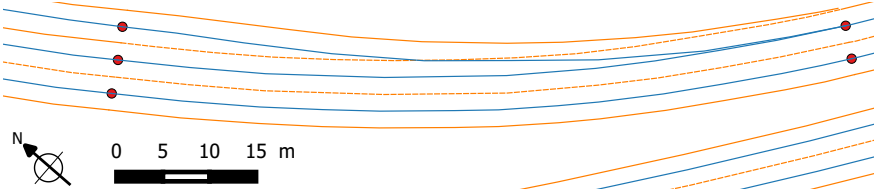

Figure 1. Detail of the map at a lane forking in Compiègne, France. The centerline of the lanes are drawn in blue and the lane markings in brown. The upper road is drivable from East to West. The upper blue polyline of the right lane separates into two lanes.

- Road Geometric Information: as for any road maps (brown lines on Fig. 1). Polylines describe the geometry of the driving lanes in a local Cartesian frame. The road network is split into Links representing a segment of road bearing the same properties (e.g. width, lane markings, etc).

- Lane Markings: each drivable lane has information on the nature of the markings delimiting it (blue lines on Fig. 1). A geometric description is given and additional information are included such as the type of marking (e.g. solid line).

- Road Connectedness: to navigate in the Links network, information about connected links (i.e. previous and next accessible links) is given. This gives a more efficient way to determine which link is reachable along the track of the current position (respecting traffic rules) without costly distance calculation.

- Road Adjacency: as the previous property, this allows determining if there are lanes on the side of the current one available form cross-track evolution, i.e. lane changing.

All these pieces of information are available easily and at low computing cost for any Link in the map, being a relational database. The filter relies on this to perform efficiently.

\section{B. Map-matching Integrity}

A map-matching respects an integrity level if the set of hypotheses provided as solution contains the correct one, with respect to a given risk. In other words, an algorithm with a risk of $10^{-2}$ (i.e. $1 \%$ ) has to return the actual map-matched position (i.e. the matching ground truth) $99 \%$ of the time.

Applied to an HD map described by polylines as the one used in this paper, as the chosen elementary geometrical descriptor is a segment (a link is a list of connected segments, each delimited by two shapepoints), the map-matched segment of the ground truth of the position has to part of the set of hypotheses returned by the MM algorithm, according to the given risk.

\section{Particle Filter}

The developed map-matching method is based on a Particle Filter (PF). This method is commonly used for this purpose [6], [7], [8], but the integrity of the result is more rarely discussed. PF's nature to manage multiple hypotheses is interesting in this context to provide a certain level of integrity, especially in ambiguous situations, where single hypothesis methods could lose track of the correct solution. The described
PF implements measures to avoid this situation by exploring and keeping the maximum number of likely hypotheses.

1) Car State Model: The car is modeled by a 2D pose $X_{p}^{i}$ (composed of the Cartesian 2D coordinates $\left(x^{i}, y^{i}\right)$ and heading $\psi^{i}$ ) and the ID of the map link it is matched to $\left(m l^{i}\right)$ as described by Eq. 1 . This is the state $X^{i}$ of each particle that represents a single position hypothesis with its map-matched solution.

$$
X^{i}=\left(X_{p}^{i}, m l^{i}\right)=\left(x^{i}, y^{i}, \psi^{i}, m l^{i}\right)
$$

Additionally, each particle possesses a weight $w^{i}$ characterizing its likelihood as a matching solution. The complete structure of a particle is then given by Eq. 2:

$$
\operatorname{Part}^{i}=\left(X^{i}, w^{i}\right)
$$

2) Filter Principle: PF map-matching relies on the intrinsic multi-hypotheses nature of the filter. Combined with the properties of the map described in Sec. II-A, a throughout search for the best matching hypothesis can be performed efficiently. While each particle is associated to a given link (step done during the initialization of the filter), it evolves freely on the $2 \mathrm{D}$ plan, and is not only constrained to the link itself. This gives more freedom to the particles, allowing a better exploration of the map (e.g. with possible lane changes). But the matching hypothesis is also extensively used to help navigate the map data: using the road connectedness, a particle's matching easily evolves, at small computation cost.

The goal of this filter is to determine a set of particles containing the correct matching hypothesis while trying to minimize its cardinality. The filter evolution is mostly lead by dead-reckoning (DR) computed with the proprioceptive information as inputs (odometry). Thanks to a comparison between the headings of the map-matched polylines and the ones of the particles and a cross-track distance between the particle and the polyline to a lesser extent, the weights of the particles are updated which conducts the filter to eliminate diverging particles and duplicate coherent ones at each resampling stage. The expected filter's output is a set of map-matching hypotheses that correspond to the odometry information and the map. The result might not be a unique hypothesis if ambiguities exist.

3) GNSS role: The GNSS influence is kept at a minimum, and act only as a way to limit the divergence of the filter: the particles getting too far away from the GNSS position are eliminated. It thus acts as a Horizontal Protection Level (HPL) giving a confidence radius around which the true position is believed to be with a high confidence level. In practice, this HPL can be provided directly by the software of the GNSS positioning system or, when no integrity information is available, recomputed to a conservative value using the residuals of the pseudo-range measurements in a snapshot way [9] or using an history of the path of the car [1]. In practice, the GNSS position is also used at the initialization of the filter: the particles are generated around the first GNSS fix with a radius of the HPL value. 


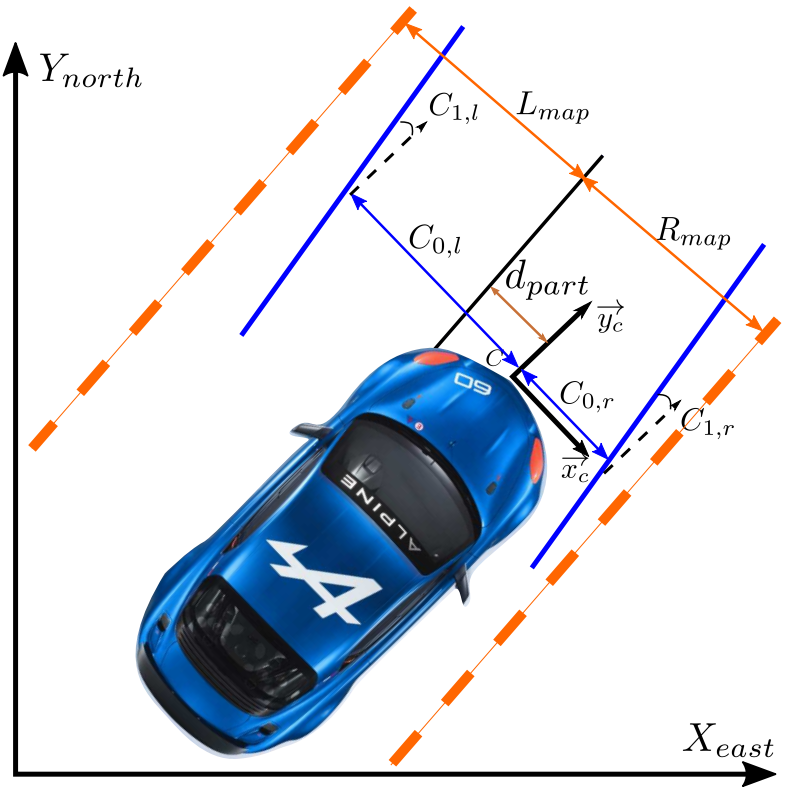

Figure 2. Frames used by the camera for lane marking description and symbols definitions: dashed orange lines represent the map's lane markings data. Camera detections are represented in blue. The lane's centerline is in black.

\section{LANE INFORMATION INTEGRATION}

The particle filter is based on the hypothesis that the car is driving on average on the center of the lane [4]. In practice, it is often not verified. This section's rationale is to enhance the filter with information from exteroceptive sensors to remove this hypothesis: lane detection from a camera system is used to determine the car's lateral position in the driving lane.

\section{A. Lane Marking Description From The Camera}

The Mobileye smart camera used in the system is able to detect the ego-lane markings and return them using polynomial models. For each detected lane marking, the camera returns the coefficients of a third degree polynomial [10] (see Eq. 3 ) approximating the equation of a clothoid, where $x$ and $y$ are coordinates in the camera working frame $\left(C, \overrightarrow{x_{c}}, \overrightarrow{y_{c}}\right)$ (see Fig. 2).

$$
y=\frac{C_{3}}{6} \cdot x^{3}+\frac{C_{2}}{2} \cdot x^{2}+C_{1} \cdot x+C_{0}
$$

In practice, the highest coefficients (the curvature $C_{2}$ and curvature derivative $C_{3}$ ) are often very noisy and do not provide reliable information. Therefore, only $C_{0}$ (the lateral offset, in meters) and $C_{1}$ (the line heading, in Rad.) are used, the lane marking being considered as a straight segment. This approximation still gives a good estimate of the line position and orientation with respect to the car. Note that the algorithm would also work with other source of information providing the lane detection in the same manner (e.g. lidar-based lane detection).

\section{B. Lane Description From The Map}

As presented in Sec. II-A, lane markings are additional information included in the map. They follow the same ge- ometric representation as the centerlines (i.e. polylines). Each driving lane references directly the lane markings delimiting it (if they exist), it is therefore possible to retrieve the equation $y=C_{1} \cdot x+C_{0}$ of the current left and right markings.

\section{Likelihood Calculation}

These exteroceptive information are mainly used during the update step of the filter, to calculate the likelihood of each particle. Note that the lane markings are not always detected by the camera. This can be caused by difficult conditions for the sensor, for instance, bad lighting, wet road, faded markings, etc. The camera also performs best when in straight lines, the marking tracking being challenging in highcurvature roads [11]. In these cases, the camera indicates a low confidence level and the likelihood calculation falls back to the "driving on the centerline" hypothesis.

If markings detection is available, lateral positioning is taken into account. The same procedure as the original filter is followed but slightly modified: previously, the likelihood followed a Gaussian function centered on the centerline; with marking information, it will be centered on the relative lateral positioning according to the detection. To do this, two position ratios are computed, describing the lateral position of the vehicle in its lane.The first uses the marking detection from the camera, i.e. the signed distances $C_{0, l}$ and $C_{0, r}$ (respectively the $C_{0}$ coefficients of the left and the right lane marking detection, and respectively negative and positive) and is defined as:

$$
r_{c a m}^{l a t}=\frac{C_{0, l}}{C_{0, l}-C_{0, r}} .
$$

- The second corresponds to the map data: for each particle (i.e. position hypothesis) the theoretical ratio is computed using $L_{\text {map }}$ and $R_{\text {map }}$ the distances of the left and right lane marking to the centerlane (both unsigned) and $d_{\text {part }}$ the signed distance of the particle to the centerlane (positive to the right):

$$
r_{\text {map }}^{\text {lat }}=\frac{L_{\text {map }}+d_{\text {part }}}{L_{\text {map }}+R_{\text {map }}} .
$$

The car within the limits of the lane markings will thus have a ratio between 0 , when on the left-side marking and 1 , on the right-side. Lane markings distances are not used in a absolute fashion in order to cope with a possible scaling factor discrepancy between the lane detection provided by the camera and the map information. In other words, if an incorrect lane width is stored in the map, the ratios are still valid. The closest to zero the value is, the better the particle fits the camera observation. The physical meaning of these ratio are a percentage of the driving lane width. For example a ratio of 0.1 is $10 \%$ of the width of a lane, e.g. on a $3.5 \mathrm{~m}$ wide lane, it represents a distance of $0.35 \mathrm{~m}$.

If the camera observations are in accordance with the particle position on the map, the two ratios will be fairly identical. To verify this, the difference between them is used to compute the likelihood:

$$
\Delta r=r_{m a p}^{l a t}-r_{c a m}^{l a t}
$$


The likelihood function is then set as a Gaussian distribution centered on 0 (i.e. a null difference meaning $r_{\text {map }}^{\text {lat }}=r_{\text {cam }}^{l a t}$, a perfect fit), described by $\mathcal{N}\left(0, \sigma_{d i s t}\right)$, with $\sigma_{d i s t}=0.1$. This difference of ratio would correspond for instance to a $35 \mathrm{~cm}$ distance on a $3.5 \mathrm{~m}$ large lane.

In the same way, instead of considering the car being aligned with the road segment, the heading information from the camera $C_{1, r}$ and $C_{1, l}$ are used to calculate the particle's heading likelihood: the mean heading $C_{1}$ is computed and serves as a reference to the particle's heading. The heading likelihood is described by $\mathcal{N}\left(0, \sigma_{\text {head }}\right)$ with $\sigma_{\text {head }}=15^{\circ}$, set empirically.

With exteroceptive lateral information, the likelihood function follows the lateral movement of the vehicle, therefore containing the particle spread in this direction. This gives a much better lateral distribution (tighter) and even allows naturally the lane changing maneuvers. Moreover it allows a better description of the car's path in the carriageway. For instance, if the vehicle drives one meter away of the center of the lane, the particles fitting this behavior will have the best likelihood score.

\section{MAP AIDED HORIZONTAL UNCERTAINTY LEVEL}

The Map-Aided HUL (MA-HUL) has been developed in [3] and is succinctly described in this section. It develops an metric used to characterize the integrity of the position of provided by the system. Its computation relies on the properties of the PF map-matching described in Sec. II: the matched hypotheses set is used to determine the level of uncertainty.

\section{A. Horizontal Uncertainty Level (HUL)}

HUL is an uncertainty metric originating from the aeronautic field. It is used to characterize the uncertainty that a GNSS position bears, given some current residuals. It takes into account several parameters including, for instance, the geometrical configuration of the satellites. It is used in conjunction with other integrity metric such as Horizontal Protection Level (HPL) and Horizontal Alarm Level (HAL) to characterize the integrity of the positioning information. To do this, classical methods need to have access to raw measurements from the GNSS receiver (pseudo-ranges), which are not available in this study.

\section{B. Map-Matching As An Independent Source Of Information}

The PF map-matching described in Sec. II is used in order to cope with the absence of pseudo-range measurement. The map-matching algorithm is designed to be as independent as possible from the GNSS (only used to prune very unlikely map-matching hypotheses), It thus acts as another independent source of positioning and provides redundancy allowing an estimation of the GNSS fix deviation with respect to the mapaided PF.

An estimate of the uncertainty level of the position is then the distance of the GNSS fix to the matching hypotheses. Fig. 3 shows an example of MA-HUL: the GNSS trace is

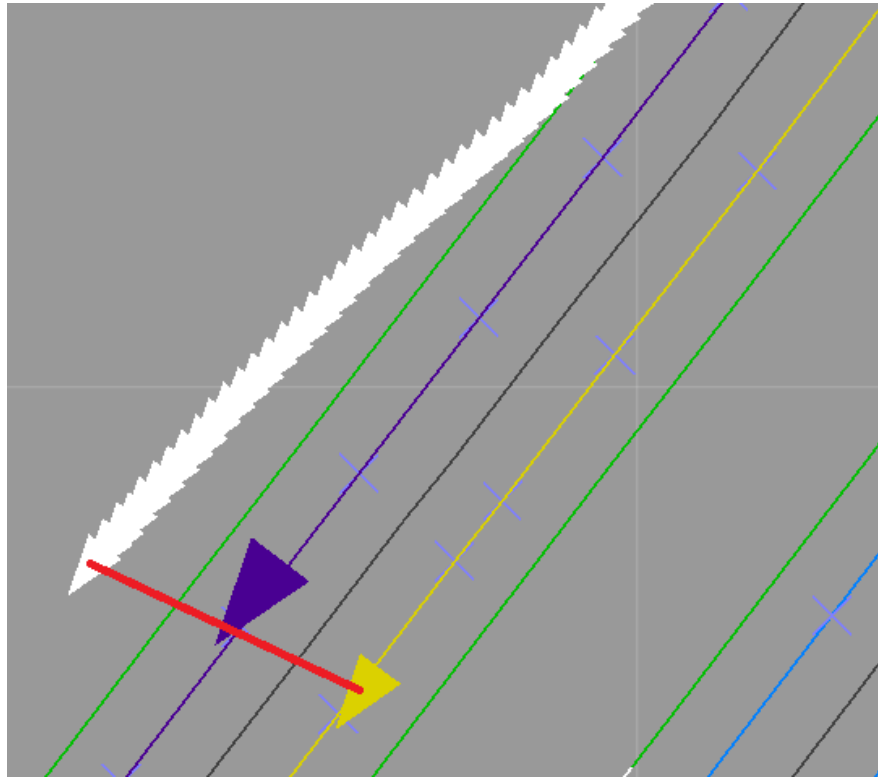

Figure 3. Biased GNSS position and associated matching hypotheses (purple and yellow triangle/line) at the same time index (the GNSS track is displayed in white). The MA-HUL is denoted by the red line, representing the largest distance between the GNSS position and a matching hypothesis. The green lines are the lane markings information from the map.

represented in white, while two matching hypotheses are in purple and yellow; the MA-HUL is represented by the red line. Note that MA-HUL is based on the assumption that the map is accurate. To keep a conservative level of uncertainty, the method chooses the farthest hypothesis for the MAHUL calculation thus considering the worst case scenario (for instance, the yellow one in Fig. 3). The computation is then given by:

$$
\text { MA-HUL }=\max _{j}\left\|X_{g n s s}-X_{h y p_{j}}\right\|^{2}
$$

The interpretation of the meaning of the MA-HUL is as follows:

- Large MA-HUL: this can be due to an erroneous GNSS fix, to an uncertain MM, to a faulty MM or any combination of these phenomenons. No confidence has to be placed in the positioning information.

- Small MA-HUL: this is the ideal situation meaning the MM result is compact and coherent with the GNSS fix. High confidence can be placed in the positioning information.

Please note that we suppose that the probability of having a faulty GNSS fix at the same position as a faulty MM is negligible.

\section{CROSS-TRACK AND ALONG-TRACK SEPARATION}

This paper improves the method presented in [3] by adding the lateral information from the camera sensor. Considering this, the particles are expected to spread differently longitudinally (along-track direction) and laterally (cross-track). The 


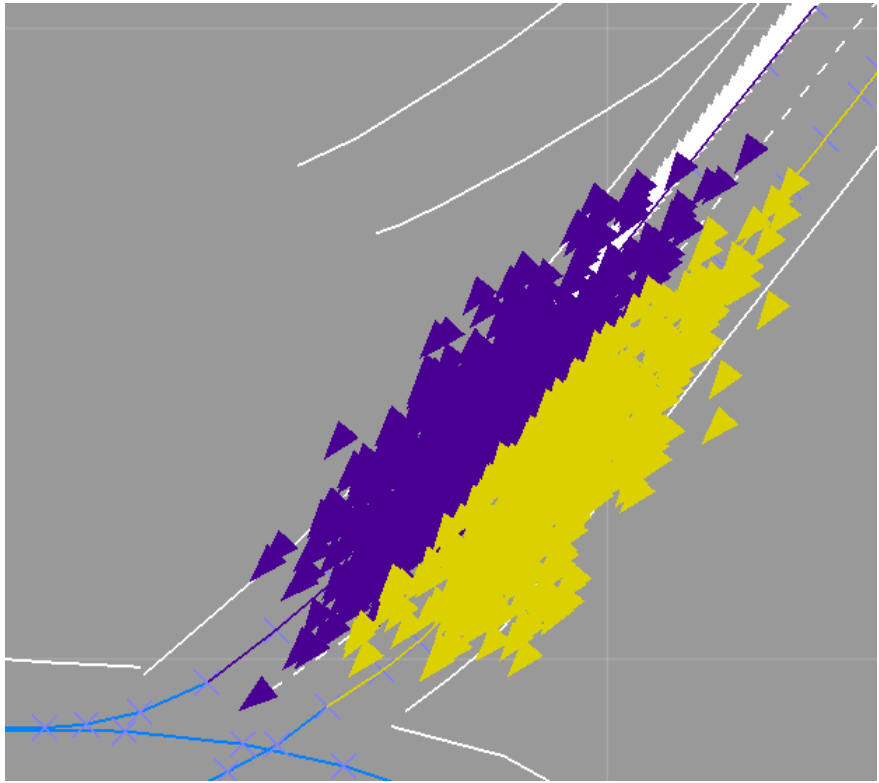

Figure 4. Filter's particles: it is clearly visible that the longitudinal spread is different from the lateral one, due to the straightness of the road driven.

phenomenon is illustrated in Fig. 4 the road leading to this area is quite straight. In this particular situation, the along-track spread is about twice as big as the cross-track. This is due to the lack of longitudinal information, the only source being the variation of the road curvature: the along-track spread is greatly reduced inside bends.

From this statement, the idea of separating the MA-HUL along the two directions emerges. The goal is to be able to characterize more finely the position uncertainty. Additionally, it gives a more tailored estimation as it targets only a specific direction: most applications are focusing on only one direction. For instance, Lane Centering Assistance (LCA) systems only need cross-track positioning. The direction-separated MAHUL will be more tied to the map-matching results than the previous combined MA-HUL, that was more general: the two directions being relative to a matched link.

An adaptation of the previous MA-HUL calculation is needed: the goal is to decompose the calculated distance into longitudinal and lateral components, with respect to the road. In other words, a change of frame has to be performed from the East-North frame to a Frenet-like, Tangent-Normal frame of the road, centered on the most likely position. Within this new frame, the coordinates $(X, Y)$ of the hypotheses will be the longitudinal and lateral components of the separated MAHUL. Homogeneous coordinates are used in order to describe the frame transformation. Let $\alpha$ be the angle formed by the road segment with the East axis. $X$ denotes the homogenous coordinates of the hypothesis in the East-North frame and $X^{\prime}$ in the new frame and finally $\left(x_{g n s s}, y_{g n s s}\right)$ are the Cartesian coordinates of the GNSS position. The transition matrix, corresponding to a translation of $\left(x_{g n s s}, y_{g n s s}\right)$ and a rotation

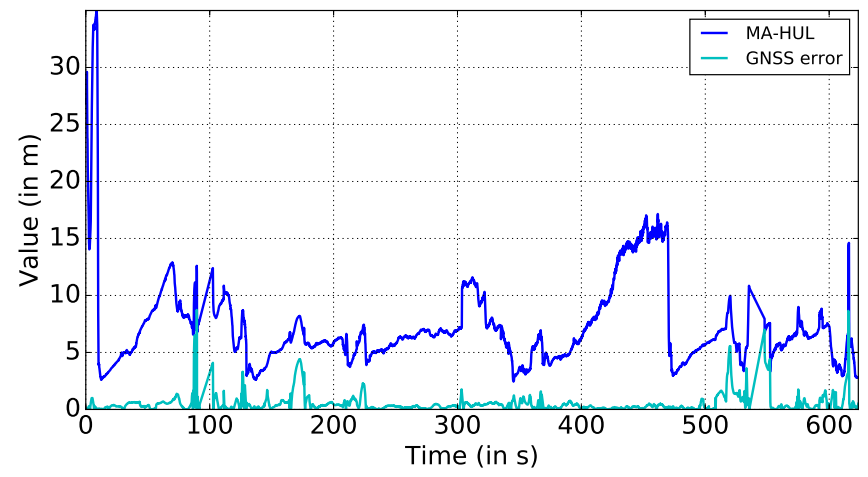

Figure 5. GNSS deviation, computed by comparing with a ground truth (in cyan) and the corresponding computed MA-HUL (in blue).

of angle $\alpha$, is then $\operatorname{Tr}$ such as:

$$
\begin{aligned}
X^{\prime} & =\operatorname{Tr} \cdot X=\left[\begin{array}{cc}
R^{T} & -R^{T} \cdot t \\
0 & 1
\end{array}\right] \cdot X \\
{\left[\begin{array}{c}
x_{\text {longi }} \\
y_{\text {lat }} \\
1
\end{array}\right] } & =\left[\begin{array}{ccc}
\cos \alpha & \sin \alpha & A \\
-\sin \alpha & \cos \alpha & B \\
0 & 0 & 1
\end{array}\right] \cdot\left[\begin{array}{c}
x_{\text {east }} \\
y_{\text {north }} \\
1
\end{array}\right] \\
A & =-\left(x_{\text {gnss }} \cdot \cos \alpha+y_{\text {gnss }} \cdot \sin \alpha\right) \\
B & =-\left(-x_{\text {gnss }} \cdot \sin \alpha+y_{\text {gnss }} \cdot \cos \alpha\right) .
\end{aligned}
$$

Once the transition done, the along-track and cross-track MA-HUL are computed by the weighted mean of the particles $X^{\prime}$ poses: the $x_{\text {longi }}$ coordinate corresponding to the alongtrack MA-HUL and $y_{\text {lat }}$ to the cross-track.

\section{RESULTS}

The algorithm has been tested with real data gathered on open road using the Papcus Framework (developed in-house by Heudiasyc) that provides data recording and real-time replay. Thanks to a development in $\mathrm{C}++$, the $\mathrm{PF}$ runs in real-time with 1000 particles. The map-matching ground truth has been created by hand labeling the GNSS position, using a context camera fixed behind the windscreen of the car. The HPL is set manually to a conservative value of $50 \mathrm{~m}$.

The test travel is representative of a peri-urban trip: the car traveled $4 \mathrm{~km}$ on 2-lanes roadways, crossing 3 roundabouts. At some point, the road gets close to high-rise buildings, creating an area prone to GNSS multi-path. On the other hand, the travel was done on a small bypass in open sky condition during $2 \mathrm{~km}$.

Fig. 5 shows the MA-HUL (in blue) computed without cross/along-track separation nor lane markings information. The convergence of the filter is about 10s after initialization. The MA-HUL is, as expected, an upper bound of the GNSS error (in cyan), but its value is in some situation quite pessimistic (e.g. the spike at around $t=460 \mathrm{~s}$ ).

Fig. 6 shows the MA-HUL with along/cross-track separation (respectively in cyan and green). This allows the identificaion of the main component of the HUL: it is visible that the variations observed in the combined MA-HUL (in blue) are 
Table I

STANDARD DEVIATION AND MEAN VALUE OF THE DIFFERENT MA-HUL CALCULATION METHODS.

\begin{tabular}{l|c|c} 
(values in meters) & Mean Value & Standard Deviation \\
\hline Combined MA-HUL & 7.23 & 3.97 \\
\hline Longitudinal MA-HUL & 3.74 & 2.32 \\
Lateral MA-HUL & 4.01 & 2.96
\end{tabular}

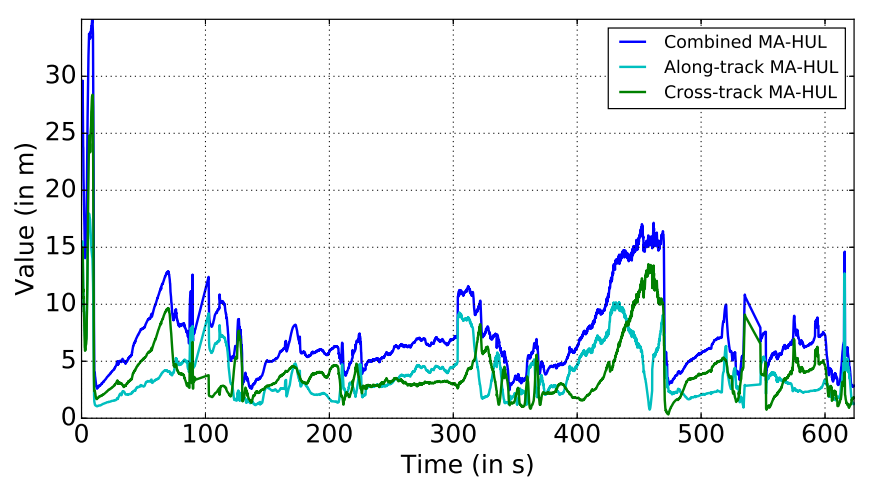

Figure 6. Separating the MA-HUL in two: along-track (in cyan) and crosstrack (in green). The camera detections are used, improving the cross-track uncertainty.

often mainly due to only one direction-separated MA-HUL, depending on the situation. For instance, at $t=60 \mathrm{~s}$ the crosstrack uncertainty causes the majority of the HUL value. This is due to a lane bifurcation on the leftmost of the road: an immediate increased is observed and a higher spread occurs whereas the longitudinal spread increases only steadily.

Another point of interest is the sequence at around $t=$ $400 s$. It also describes a lane forking (depicted in Fig. 1), but the road observes later a high curvature. The hypothesis creation is visible by an initial high increase of cross-track uncertainty. Hypotheses eliminations are visible at $t=455 \mathrm{~s}$ and $t=470 \mathrm{~s}$ by a sudden drop. Meanwhile the along-track uncertainty evolves normally in $t=[400 ; 425] \mathrm{s}$. A progressive decrease can then be observed due to the curvature that tighten the longitudinal uncertainty. It then evolves according to a particle filter behavior.

Table I compares the performance of the separated MAHUL and the combined one. It confirms the trend of Fig. 6 that separating the MA-HUL on two axis (longitudinal and normal, in a Frenet-like frame) gives lower values of uncertainties. Separating the two is therefore beneficial especially for specialized applications that only need one of the two dimensions. For instance, for lateral lane determination, this method gives $10 \%$ of the time a MA-HUL value smaller than $1.75 \mathrm{~m}$ (half a drivable lane width in France) and $57 \%$ of the time smaller than $3.5 \mathrm{~m}$.

\section{CONCLUSION}

The work presented in this paper aims to quantify the confidence a navigation system can have in map-matched estimates by giving a reliable uncertainty indicator. The solution is based on independent map-aided dead-reckoning that provides an efficient way to estimate in real-time an uncertainty bound of the GNSS positioning error, needed for ADAS or autonomous navigation functions which require high integrity positioning. The proposed MA-HUL estimates the level of uncertainty of the GNSS position, given the inputs from other sources of information - namely a map-matching algorithm based on dead-reckoning, camera lane detection and an HD road map. It counterbalances the absence of pseudorange measurements through the processing of other GNSSindependent information sources to provide redundancy to the positioning system.

In the paper, we have also described the way to separate the along-track and cross-track components of the MA-HUL which is useful to determine more accurately the uncertainty characteristics. For instance, some functionalities may only need the uncertainty in one direction. The separated MA-HULs are about half the value of the combined MA-HUL. Moreover, experimental results show that, with a low cost GNSS receiver, the cross-track MA-HUL can be $10 \%$ of the time smaller than half a lane width $(1.75 \mathrm{~m})$ when the combined one can only attain $2.4 \mathrm{~m}$. This illustrates well the improvement made by partitioning the MA-HUL in two directions.

Further work shall integrate probabilistic techniques to obtain an integrity metric corresponding to a specified risk level for map-relative localization.

\section{REFERENCES}

[1] O. Le Marchand, P. Bonnifait, J. Ibanez-Guzman, and D. Betaille, "Vehicle localization integrity based on trajectory monitoring," in IEEE/RSJ International Conference on Intelligent Robots and Systems IROS, 2009, pp. 3453-3458.

[2] O. Le Marchand, P. Bonnifait, J. Ibañez-Guzmán, F. Peyret, and D. Betaille, "Performance Evaluation of Fault Detection Algorithms as Applied to Automotive Localisation," in European Navigation Conference - GNSS 2008, Apr. 2008.

[3] F. Li, P. Bonnifait, and J. Ibanez-Guzman, "Using High Definition Maps to Estimate GNSS Positioning Uncertainty," in European Navigation Conference 2017, May 2017.

[4] F. Li, P. Bonnifait, J. Ibanez-Guzman, and C. Zinoune, "Lane-level map-matching with integrity on high-definition maps," in 2017 IEEE Intelligent Vehicles Symposium (IV). IEEE, jun 2017, pp. 1176-1181.

[5] Jie Du and M. Barth, "Next-Generation Automated Vehicle Location Systems: Positioning at the Lane Level," IEEE Transactions on Intelligent Transportation Systems, vol. 9, no. 1, pp. 48-57, mar 2008.

[6] F. Gustafsson, F. Gunnarsson, N. Bergman, U. Forssell, J. Jansson, R. Karlsson, and P.-J. Nordlund, "Particle filters for positioning, navigation, and tracking," IEEE Transactions on Signal Processing, vol. 50, no. 2, pp. 425-437, 2002.

[7] J. Levinson, M. Montemerlo, and S. Thrun, "Map-Based Precision Vehicle Localization in Urban Environments," in Robotics: Science and Systems III. Robotics: Science and Systems Foundation, jun 2007.

[8] J. Rabe, M. Necker, and C. Stiller, "Ego-lane estimation for lanelevel navigation in urban scenarios," in 2016 IEEE Intelligent Vehicles Symposium (IV). IEEE, jun 2016, pp. 896-901.

[9] J. Cosmen-Schortmann, M. Azaola-Saenz, M. A. Martinez-Olague, and M. Toledo-Lopez, "Integrity in urban and road environments and its use in liability critical applications," in 2008 IEEE/ION Position, Location and Navigation Symposium, May 2008, pp. 972-983.

[10] Z. Tao, P. Bonnifait, V. Fremont, and J. Ibanez-Guzman, "Mapping and localization using GPS, lane markings and proprioceptive sensors," in 2013 IEEE/RSJ International Conference on Intelligent Robots and Systems. IEEE, nov 2013, pp. 406-412.

[11] Z. Kim, "Robust Lane Detection and Tracking in Challenging Scenarios," IEEE Transactions on Intelligent Transportation Systems, vol. 9, no. 1, pp. 16-26, mar 2008. 